\title{
Estudio de la emisión de radiación de frenado mediante simulaciones mixtas con el código Monte Carlo PENELOPE
}

\section{Study of the Bremsstrahlung emission through mixed simulations with Monte Carlo code PENELOPE}

\author{
Pedro Antonio García Higueras, Salvador García Pareja, Francisco Javier Casado Villalón \\ Hospital Universitario Regional de Málaga Avda. Carlos Haya- 29010 Málaga.
}

Fecha de Recepción: 03/03/2020 - Fecha de Aceptación: 09/07/2020

El método Monte Carlo permite la descripción precisa de las propiedades de la radiación de frenado, la cual se emplea en medicina para radiodiagnóstico o radioterapia. El código PENELOPE descansa en la implementación de historias condensadas para el transporte de partículas cargadas y permite al usuario adaptar el grado de detalle de las simulaciones mediante los parámetros de simulación. La variación de estos parámetros puede afectar a las distribuciones de partículas obtenidas al simular radiación de frenado producida en blancos gruesos.

En este trabajo se analizan las diferencias entre simulaciones con distinto grado de detalle mediante la reproducción con PENELOPE de experimentos publicados de producción de radiación de frenado con electrones de alta y baja energía. Para ello se varían los parámetros de simulación $C_{1}$ y $C_{2}$ hasta alcanzar un valor a partir del cual los resultados de las simulaciones son estadísticamente equivalentes.

Se ha observado que una inadecuada elección de los parámetros de simulación conlleva una reproducción inexacta de las características del haz de frenado. Se necesita mayor grado de detalle para simular electrones de alta energía en un material de alto número atómico que para electrones de baja energía en un material de bajo número atómico.

Palabras clave: Bremsstrahlung, simulaciones mixtas, Monte Carlo, PENELOPE.

The Monte Carlo method allows the accurate description of the properties of a bremsstrahlung radiation, which is used in medicine for radiodiagnosis or radiotherapy. PENELOPE code is based on the condensed histories implementation for charged particles transport mechanics and allows the user to adapt the simulation detail through simulation parameters $C_{1}$ and $C_{2}$. The variation of these parameters can affect the particle distributions obtained simulating bremsstrahlung beams from thick targets.

In this work has been analyzed the differences between simulations with different values of simulation parameters through reproduction with PENELOPE of published experiments of bremsstrahlung production with high and low energy electrons. Simulation parameters $C_{1}$ and $C_{2}$ have been varied until reaching a value from which the simulations results are equivalent within the statistical uncertainty considered.

The use of large simulation parameters values for condensed simulation of electrons in bremsstrahlung production conducts to particles fluence deviations from experimental published results, especially on the central axis. More detailed simulation within the target is needed with high energy electrons in a high atomic number material than low energy electrons in a low atomic number material.

Key words: Bremsstrahlung, mixed simulations, Monte Carlo, PENELOPE.

\footnotetext{
*Correspondencia: pantonio.garcia.sspa@juntadeandalucia.es https://doi.org/10.37004/sefm/2020.21.2.002
} 


\section{Introducción}

La radiación de frenado (o bremsstrahlung) se compone de rayos $X$ generados por la desaceleración de partículas cargadas en materia. Su aplicación en medicina comprende un amplio rango de energías, desde las decenas de keV en radiodiagnóstico hasta los $\mathrm{MeV}$ en terapia. En este ámbito, los rayos $X$ se producen al frenar electrones acelerados en blancos gruesos metálicos. La descripción pormenorizada tanto del espectro energético como de la distribución angular de los fotones de frenado permite cálculos precisos en la dosis recibida por el paciente, y representa una descripción rigurosa de la calidad del haz de radiación.

El código de transporte de partículas PENELOPE ${ }^{1}$ es un conjunto de programas y subrutinas escritos en FORTRAN 90 que emplean el método Monte Carlo (MC) para modelar el transporte acoplado de fotones, electrones y positrones en un rango energético que abarca desde unas centenas de eV hasta $1 \mathrm{GeV}$.

Una simulación se denomina detallada cuando se reproducen todas y cada una de las interacciones de una partícula hasta que es absorbida. Debido al gran número de interacciones que una partícula cargada puede llegar a experimentar antes de ser absorbida, los códigos MC de propósito general descansan en la implementación de técnicas de historias condensadas para el transporte de partículas cargadas empleando teorías de dispersión múltiple. Estas técnicas consisten en resumir un gran número de eventos blandos en un único evento artificial entre dos eventos duros, lo que permite reducir el tiempo de simulación.

A medio camino entre las simulaciones detalladas y las puramente condensadas se encuentran las simulaciones mixtas. El código PENELOPE posibilita la modificación del grado de detalle de las simulaciones en el transporte de electrones y positrones mediante el ajuste por parte del usuario de los parámetros de simulación.

Las ventajas de las simulaciones mixtas sobre las completamente condensadas es que ofrecen una mayor precisión en la simulación de partículas cargadas, especialmente en las cercanías de separación entre medios. Al disminuir el valor de los parámetros de simulación mixta, aumenta el grado de detalle de la simulación pero también el tiempo necesario para simular cada historia.

Algunos autores ${ }^{2,3}$ han demostrado que al simular radiación de frenado producida en blancos gruesos la elección de los parámetros de simulación puede afectar a los resultados obtenidos. Otros autores ${ }^{4,5}$ han evaluado la influencia de la energía inicial del haz de electrones y el número atómico $(Z)$ del blanco en la exactitud de las propiedades de un haz de radiación de frenado caracterizado por MC.
El objetivo de este trabajo es estudiar la dependencia de los resultados obtenidos en las simulaciones con los parámetros de simulación mixta. Se va a reducir el valor de estos parámetros hasta que los resultados de las simulaciones sean estadísticamente equivalentes. Estos resultados van a ser cotejados con datos experimentales publicados. Para evaluar el efecto del número atómico y la energía del haz se van a reproducir dos experimentos: el primero de un haz de electrones de $15 \mathrm{MeV}$ impactando en un blanco de plomo $(\mathrm{Pb})$ y un segundo de electrones de $200 \mathrm{keV}$ en un blanco de aluminio (Al).

\section{Materiales y métodos}

\section{PENELOPE}

Las simulaciones han sido realizadas con la versión 2014 del programa en un equipo con el sistema operativo Windows 10 de 64 bits y una CPU Intel(R) Core (TM) $17-7700 \mathrm{HQ}$ de $2.8 \mathrm{GHz}$.

PENELOPE ha sido validado en la reproducción de espectros de fotones de bremsstrahlung por Salvat et al. ${ }^{6}$ La sección eficaz diferencial de bremsstrahlung se obtiene de datos publicados. ${ }^{7,8}$ La modelización de la distribución angular de los fotones generados está basada en un ajuste semi-empírico que para electrones con una energía menor a 500 keV emplea la función de ajuste de onda parcial de Kissel et al. ${ }^{9}$ y para electrones de energía superior es aproximada por una distribución dipolar clásica. Las colisiones inelásticas individuales de las partículas cargadas se describen con los datos de sección eficaz diferencial publicados en el ICRU Report No. 77. ${ }^{10}$ Una descripción más detallada de los modelos empleados puede ser encontrada en el manual del programa. ${ }^{11}$

Los parámetros de simulación mixta que tienen dependencia en el grado de detalle de la simulación son $C_{1}, C_{2}, W_{c c}$ y $W_{c r}$.

- $C_{1}$ determina el ángulo de corte $\theta_{c}$ utilizado para clasificar los eventos elásticos en duros 0 blandos. Sus valores pueden variar entre 0.2 (simulación condensada) y 0.0 (simulación completamente detallada).

- $\quad C_{2}$ es la fracción de energía máxima perdida en un único paso. Sus valores pueden variar entre 0.2 y 0.0 .

- $\quad W_{c c}$ es el valor de corte para pérdidas energéticas en colisiones inelásticas. Las interacciones con pérdidas energéticas menores a $W_{c c}$ se consideran interacciones blandas. 
- $\quad W_{c r}$ es el valor de corte para emisión de bremsstrahlung. Las interacciones que producen emisiones de fotones con energías menores a $W_{c r}$ se consideran interacciones blandas.

En los dos experimentos reproducidos se van a realizar diferentes simulaciones mixtas que progresivamente se van aproximando a una simulación completamente detallada mediante la reducción de los parámetros de simulación mixta $C_{1}$ y $C_{2}$ de manera que en cada simulación $C_{1}=C_{2}$ tal y como se recomienda en el manual del programa.

Las incertidumbres en los resultados de las simulaciones se representan con un factor de cobertura $k=2$ considerando sólo incertidumbres estadísticas.

La variación de tiempo que se produce al disminuir el valor de los parámetros de simulación mixta se va a evaluar con el concepto de eficiencia ${ }^{11} \varepsilon$ :

$$
\varepsilon=\left(\frac{\bar{x}}{s_{\bar{x}}}\right)^{2} \cdot\left(\frac{1}{T}\right)
$$

donde $\bar{x}$ es el valor medio de la distribución de valores, $s_{\bar{x}}$ es la varianza asociada a $\bar{x}$ y $T$ el tiempo de simulación.

\section{Experimento 1: Electrones de $15 \mathrm{MeV}$ en plomo}

El primero de los experimentos reproducidos es el realizado por Faddegon et al. ${ }^{12-13}$ en un acelerador lineal Vickers del National Research Council of Canada.
En el experimento se emplea un haz de electrones de $0.35 \mathrm{~cm}$ de diámetro con una incidencia normal a una ventana de titanio, para posteriormente acceder, atravesando una cavidad de aire y un monitor de transmisión de corriente (MTC), a un blanco grueso donde se va a producir la práctica totalidad de la radiación de frenado. Se ha empleado una ventana de acero inoxidable (Al) tras el MTC en las medidas de ángulos pequeños, comprendidos entre $0^{\circ}$ y $10^{\circ}$ inclusive. Para ángulos grandes, comprendidos entre $30^{\circ}$ y $90^{\circ}$, la ventana de Al no se ha empleado (fig. 1).

Los fotones generados son detectados por un cristal centelleador de $20 \times 25 \mathrm{~cm}^{2}$ de Nal. El detector se encuentra a una distancia de $318-361 \mathrm{~cm}$ desde la cara superior del blanco ubicado en el eje central de un colimador de $\mathrm{Pb}$ de $2.4 \mathrm{~cm}$ de diámetro y $20 \mathrm{~cm}$ de espesor.

En los resultados obtenidos se han realizado correcciones por la atenuación producida por el aire que se encuentra entre el blanco y el detector, por el efecto del colimador de plomo, apilamiento de pulsos, respuesta y eficiencia del detector.

Los resultados experimentales se muestran para la fluencia $\left(1 / \mathrm{m}^{2}\right)$ en función del ángulo en unidades de número de partículas normalizado por el área del detector $\left(\mathrm{m}^{2}\right)$ y número de partículas lanzadas al blanco. Para los espectros se emplea la fluencia energética $\left(1 / \mathrm{m}^{2}\right)$ en unidades de energía (MeV) normalizada por el área del detector $\left(\mathrm{m}^{2}\right)$, ancho de energía de la banda de medida (MeV) y número de partículas lanzadas al blanco.

Faddegon et al. ${ }^{13}$ presentan unas incertidumbres en la fluencia del $5 \%$ en el eje del haz y de hasta un

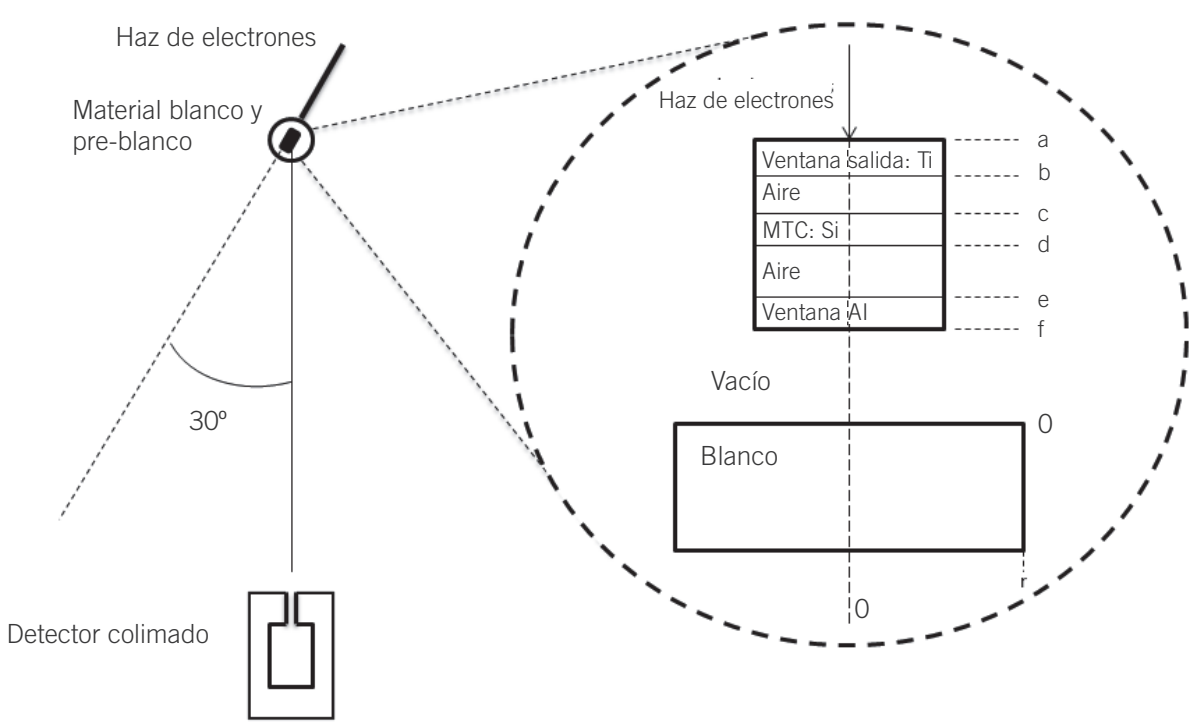

Fig. 1. Esquema de los elementos descritos en el experimento 1 para su reproducción por MC. Figura adaptada de la publicada por Faddegon et al. ${ }^{2}$ 
7\% fuera de él. En el caso de las medidas de espectros oscilan entre un $3 \%$ y un $10 \%$.

\section{Experimento 2: Electrones de $200 \mathrm{keV}$ en aluminio}

Los resultados experimentales con los que se van a comparar las simulaciones realizadas están publicados por Rester et al., ${ }^{14}$ los cuales aplican la disposición experimental descrita por Dance et al. ${ }^{15}$

El haz de electrones producido es acelerado mediante un dispositivo Van der Graff LTV 3-MeV. Una vez acelerado accede al interior de una cámara cilíndrica de aluminio en la cual se ha realizado el vacío. Esta cámara cilíndrica está conectada eléctricamente a tierra, formando alrededor del blanco una jaula de Faraday.

El haz (fig. 2) penetra en la cámara a través de un orificio de $2.54 \mathrm{~cm}$ de diámetro y $1.27 \mathrm{~cm}$ de espesor. Este mismo orificio se ha efectuado a la salida del haz de radiación, con un recorrido angular que abarca desde $-15^{\circ}$ a $160^{\circ}$. El material de la ventana permite la entrada o salida de las distintas partículas sin ser atenuadas. ${ }^{15}$

Una vez el haz emerge del dispositivo anterior, atraviesa un colimador de plomo, el cual tiene un imán cuya finalidad consiste en repeler los electrones dispersados. Finalmente los fotones de bremsstrahlung alcanzan un detector de centelleo de $\mathrm{Nal}(\mathrm{TI})$.

El detector de centelleo está rodeado por un blindaje de plomo, excepto por un pequeño orificio de apertura de $1.27 \mathrm{~cm}$ de diámetro y una altura de $7.62 \mathrm{~cm}$,

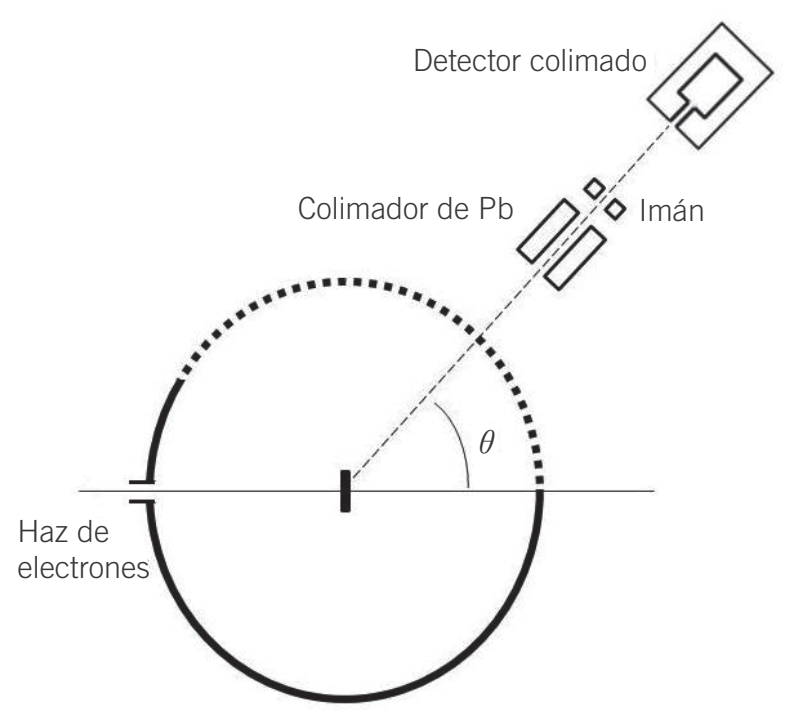

Fig. 2. Esquema de los elementos descritos en el experimento 2 para su reproducción por MC. Figura adaptada de la publicada por Dance et al. ${ }^{15}$ enfrentado en el mismo eje a una distancia de $98.1 \mathrm{~cm}$ de la cara superior del blanco. La apertura subtendida por el ángulo sólido es de $1.31 \cdot 10^{-4} \mathrm{sr}$ respecto a la posición del blanco. El soporte que sustenta el detector rota sobre unas guías en un arco que va desde $0^{\circ}$ a $150^{\circ}$ con el centro de giro en el centro de la cara superior del blanco.

El blanco utilizado es de aluminio puro de forma que el espesor se ha escogido ligeramente mayor al rango medio de los electrones de dicha energía en el material. Para todas las medidas el haz de electrones incide de forma normal a la superficie del blanco.

Los resultados experimentales se muestran para la fluencia energética $\left(\mathrm{MeV} / \mathrm{m}^{2}\right)$ en función del ángulo en unidades de energía (MeV) normalizado por el área del detector $\left(\mathrm{m}^{2}\right)$ y número de partículas lanzadas al blanco. Para los espectros se emplea la fluencia energética $\left(1 / m^{2}\right)$ en unidades de energía (MeV) normalizada por el área del detector $\left(\mathrm{m}^{2}\right)$, ancho de energía de la banda de medida $(\mathrm{MeV})$ y número de partículas lanzadas al blanco.

Las incertidumbresexperimentales de espectros y fluencias oscilan entre un $20 \%$ y un $30 \%$ y en su estimación hay muchos factores que varían en función del ángulo y la energía del haz. Más detalles están publicados por Rester et al. ${ }^{14}$

\section{Simulación 1: Electrones de 15 MeV en plomo}

Los detalles de la simulación reproducen las indicaciones dadas por Faddegon et al. ${ }^{2}$

La fuente, compuesta por electrones monoenergéticos de $15.18 \mathrm{MeV}$, se ha simulado como un haz cuadrado de $0.35 \times 0.35 \mathrm{~cm}^{2}$ de fluencia constante e incidencia normal a la ventana de titanio. La simulación de la geometría desde la ventana de titanio hasta el blanco ha sido la misma que en la disposición experimental descrita (tabla 1).

Para el blanco se ha empleado $\mathrm{Pb}$ puro con una densidad de $11.43 \mathrm{~g} / \mathrm{cm}^{3}$ y un espesor másico de $9.13 \mathrm{~g} / \mathrm{cm}^{2}$. La ventana de salida está compuesta de titanio puro de densidad $4.54 \mathrm{~g} / \mathrm{cm}^{3}$ y el MTC de silicio puro con densidad de $2.33 \mathrm{~g} / \mathrm{cm}^{3}$. El aire dentro de la cámara está a temperatura ambiente, simulado con una densidad de $0.001205 \mathrm{~g} / \mathrm{cm}^{3}$ y compuesto por C, N, $\mathrm{O}$ y $\mathrm{Ar}$ en proporciones de $0.000124,0.756,0.232$ y 0.0128 en peso. El Al tiene una densidad de $8.06 \mathrm{~g} / \mathrm{cm}^{3}$ y está compuesto por $\mathrm{C}$, Si, $\mathrm{Cr}, \mathrm{Mn}$, Fe y $\mathrm{Ni}$ en proporciones de 0.001, 0.007, 0.18, 0.01, 0.712 y 0.09, respectivamente, en peso. La región comprendida entre el blanco y el detector se ha simulado como vacío ya que las medidas han sido corregidas por el efecto del aire. El detector se ha simulado en una superficie esférica de $100 \mathrm{~cm}$ de radio centrada en el punto central de la cara superior del blanco. 
Tabla 1. Posición de los diferentes componentes utilizados en el experimento realizado por Faddegon et al. ${ }^{2}$ El origen ha sido tomado en la superficie superior del blanco de $\mathrm{Pb}$.

\begin{tabular}{c|c} 
Punto & Posición (cm) \\
\hline a & 3.313 \\
\hline b & 3.3 \\
\hline c & 2.91 \\
\hline$d$ & 2.9 \\
\hline e & 1.6051 \\
\hline$f$ & 1.6 \\
\hline$g$ & 1.583
\end{tabular}

Las energías de absorción se han establecido en $200 \mathrm{keV}$ y $10 \mathrm{keV}$ para electrones (o positrones) y fotones respectivamente. Las parámetros $C_{1}$ y $C_{2}$ se han establecido en valores de 0.2, 0.1, 0.05, 0.01 y 0.001 . El corte correspondiente a las interacciones inelásticas y de bremsstrahlung, $W_{c c}$ y $W_{c r}$ se ha establecido en $200 \mathrm{keV}$ y $10 \mathrm{keV}$, respectivamente.

Se ha realizado un escalado de la fluencia aplicando la ley del inverso al cuadrado de la distancia debido a la diferencia de la medida en la simulación $(100 \mathrm{~cm})$ y el experimento $(318-361 \mathrm{~cm})$.

\section{Simulación 2: Electrones de $200 \mathrm{keV}$ en aluminio}

En el experimento de referencia descrito por Dance et al. ${ }^{15}$ no se dan detalles de los distintos materiales y geometrías, por lo que se ha realizado una simulación en la que solo se va a considerar el blanco. La fuente, compuesta por electrones monoenergéticos de $200 \mathrm{keV}$, se ha considerado como una fuente puntual en la que los electrones son emitidos en dirección normal a la superficie del blanco en el mismo eje en el que se encuentra su centro.

Para el blanco se ha empleado Al puro con una densidad de $2.698 \mathrm{~g} / \mathrm{cm}^{3}$ y con un espesor másico de $0.069 \mathrm{~g} / \mathrm{cm}^{2}$. La región comprendida entre el blanco y el detector se ha considerado como vacío al no existir datos sobre materiales y geometría y al considerar los autores de los experimentos que no afectan a los resultados obtenidos.

El detector se ha simulado como una superficie esférica de $98.1 \mathrm{~cm}$ de radio centrada en la cara superior del banco. El ancho de energía ha sido establecido del mismo tamaño que los resultados experimentales de referencia para no añadir incertidumbres a la comparación.
Las energías de absorción se han establecido en 36 keV para fotones y electrones coincidiendo con la energía de corte experimental. Los parámetros $C_{1}$ y $C_{2}$ han sido establecidos en valores de 0.2, 0.1, 0.05 y 0.01 . El corte correspondiente a las interacciones inelásticas y de bremsstrahlung $W_{c c}$ y $W_{c r}$ se ha establecido en $36 \mathrm{keV}$.

\section{Resultados}

\section{Resultados 1: Electrones de $15 \mathrm{MeV}$ en plomo}

Los espectros energéticos de fotones por electrón incidente obtenidos han sido corregidos por el inverso al cuadrado de la distancia y normalizados al ángulo sólido subtendido por los detectores. Se ha hecho uso de la simetría esférica de la geometría para agilizar la simulación.Se han empleado $5 \cdot 10^{7}$ historias por simulación realizada.

Los espectros de bremsstrahlung experimentales junto a los calculados con la simulación más detallada, donde $C_{1}=C_{2}=0.001$, se muestran en la fig. 3 para los ángulos del detector de $1^{\circ}, 4^{\circ}, 10^{\circ}, 30^{\circ}, 60^{\circ}$ y $90^{\circ}$.

Para todos los espectros se observa una gran coincidencia entre los resultados experimentales y los calculados, excepto para los ángulos de $60^{\circ}$ y $90^{\circ}$, donde la simulación sobrestima ligeramente la fluencia energética.

Los resultados de fluencia experimentales y calculados con la simulación más detallada se muestran en la fig. 4. De nuevo se observa una gran coincidencia entre ambos.

Se han representado en la fig. 5 la fluencia energética relativa respecto a la simulación más detallada realizada con $C_{1}=C_{2}=0.001$ para el ángulo de $4^{\circ}$. Se observa una diferencia relativa mayor al aumentar el valor de los parámetros de simulación mixta. Al ser esta diferencia estadísticamente constante con la energía, la discrepancia queda explicada por una diferencia en la fluencia de fotones.

Los efectos de una incorrecta elección de los parámetros de simulación mixta se aprecian en la fig. 6 , donde se observan cambios significativos en la fluencia obtenida al variar los parámetros $C_{1}$ y $C_{2}$.

Se observan mayores discrepancias para ángulos pequeños comprendidos entre $0^{\circ}$ y $10^{\circ}$. Estas discrepancias desaparecen para ángulos grandes, comprendidos entre $30^{\circ}$ y $90^{\circ}$. La diferencia relativa de fluencia en el eje central es del $4.5 \%$ para el caso de $C_{1}=C_{2}$ $=0.1 \mathrm{y}$ del $2.5 \%$ para $C_{1}=C_{2}=0.05$. En el caso de $C_{1}=C_{2}=0.01$ los resultados son estadísticamente equivalentes a los obtenidos con $C_{1}=C_{2}=0.001$. 


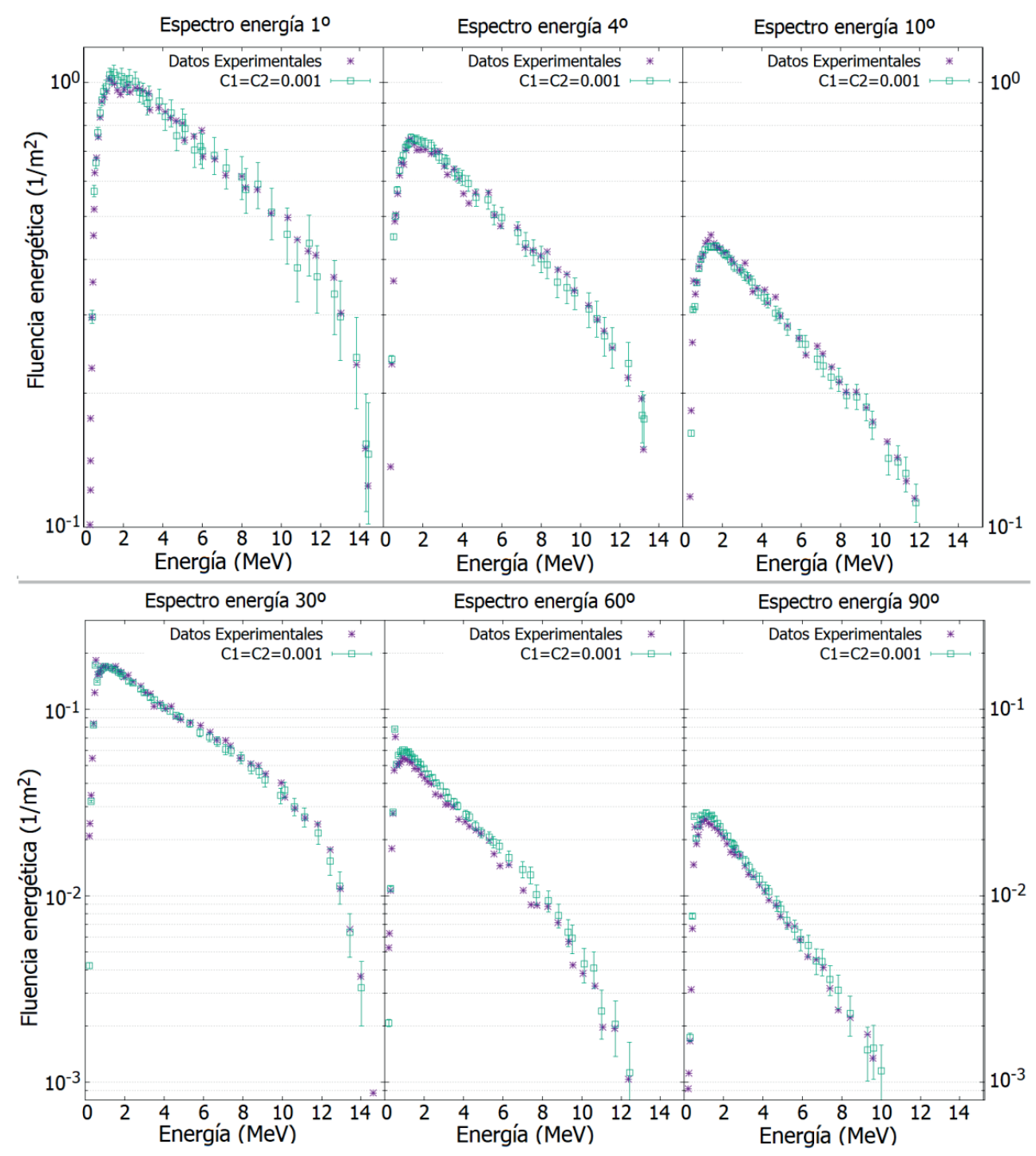

Fig. 3. Fluencia energética a 1 metro de fotones de bremsstrahlung para un haz de $15 \mathrm{MeV}$ sobre un blanco de Pb y los ángulos de $1^{\circ}, 4^{\circ}, 10^{\circ}, 30^{\circ}, 60^{\circ}$ y $90^{\circ}$. Resultados experimentales y MC con los valores de simulación mixta $C_{1}=C_{2}=0.001$.

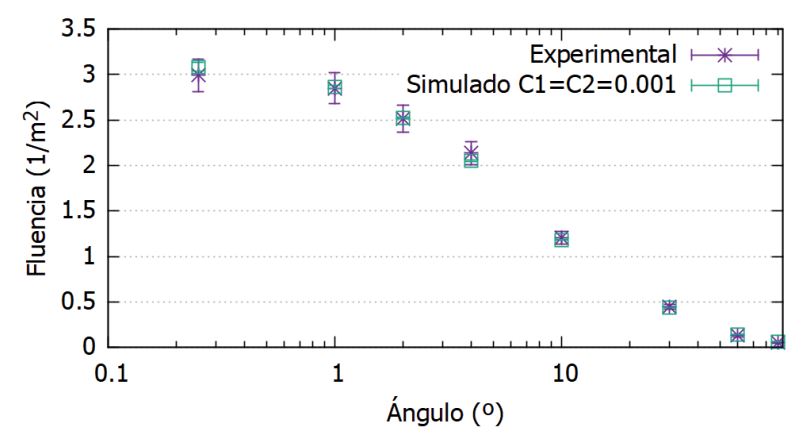

Fig. 4. Fluencia de fotones de bremsstrahlung para un haz de electrones de $15 \mathrm{MeV}$ sobre un blanco de $\mathrm{Pb}$ $(\mathrm{Z}=82)$.
Este incremento de la fluencia en el eje central al aumentar el valor de los parámetros de simulación mixta se compensa con la disminución de la fluencia a ángulos mayores. Este hecho sugiere un aumento del ángulo medio de emisión de los fotones de bremsstrahlung al disminuir los valores de $C_{1}$ y $C_{2}$. El efecto descrito desaparece con una correcta elección de los parámetros de simulación mixta.

En la tabla 2 se muestra la eficiencia (1) relativa al variar los parámetros de simulación mixta. Al disminuir los valores de $C_{1}$ y $C_{2}$ aumenta el grado de detalle de la simulación y se produce por tanto un notable aumento del tiempo de simulación para un mismo número de historias. 


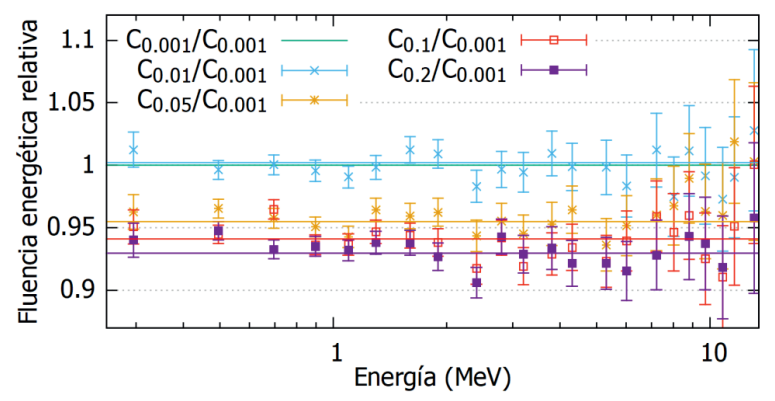

Fig. 5. Fluencia energética relativa de un haz de $15 \mathrm{MeV}$ sobre un blanco de $\mathrm{Pb}$ para el ángulo de $4^{\circ}$ respecto a la simulación más detallada realizada. Junto a los puntos se representa su valor promedio en forma de línea continua. Para facilitar la lectura se representan la mitad de los puntos y sus incertidumbres con un factor de cobertura $k=1$.

Tabla 2. Valores relativos de eficiencia de las distintas simulaciones respecto a la simulación más detallada ( $C_{0.001)}$ para electrones de $15 \mathrm{MeV}$ en $\mathrm{Pb}$.

\begin{tabular}{c|c}
$\boldsymbol{C}_{\mathbf{1}}$ y $\boldsymbol{C}_{\mathbf{2}}$ & Eficiencia relativa \\
\hline 0.001 & 1 \\
\hline 0.01 & 6 \\
\hline 0.05 & 27 \\
\hline 0.1 & 49 \\
\hline 0.2 & 84 \\
\hline
\end{tabular}

\section{Resultados 2: Electrones de $200 \mathrm{keV}$ en aluminio}

Se han empleado $7 \cdot 10^{9}$ historias en cada una de las simulaciones. Debido a la baja eficiencia en la producción de fotones de bremsstrahlung a bajas energías, la simulación con $C_{1}=C_{2}=0.001$ no ha sido incluida en el estudio.

Los espectros energéticos de fotones de bremsstrahlung experimentales junto a los calculados con la simulación más detallada, donde $C_{1}=C_{2}=0.01$, se muestran en la fig. 7 para los ángulos del detector publicados: $0^{\circ}, 30^{\circ}, 45^{\circ}, 60^{\circ}, 120^{\circ}$ y $150^{\circ}$.

Considerando la alta incertidumbre de los resultados experimentales se observa coincidencia entre los resultados experimentales y los simulados. Respecto a las simulaciones, se obtiene una mayor incertidumbre que en el experimento anterior porque a bajas energías la sección eficaz diferencial de bremsstrahlung es varios órdenes de magnitud inferior.

Los resultados de fluencia energética experimentales y calculados con la simulación más detallada se

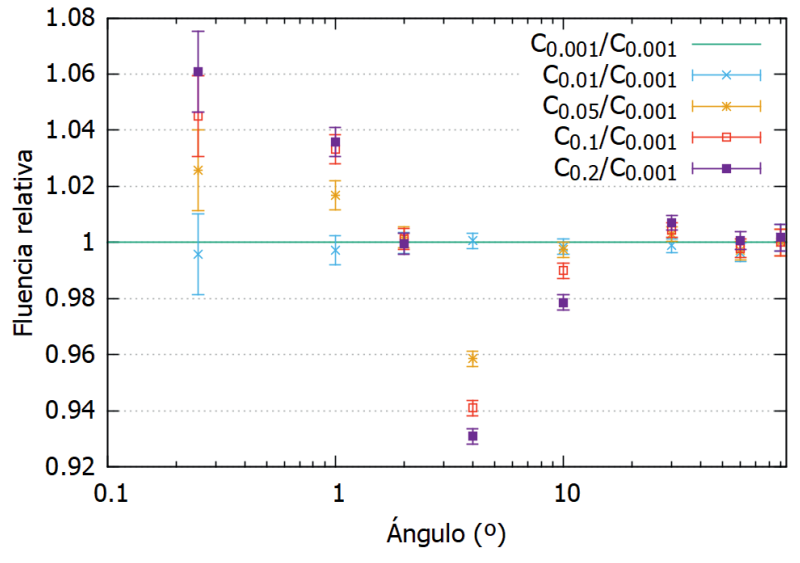

Fig. 6. Cociente entre la fluencia en cada ángulo en una simulación respecto al resultado obtenido con la simulación más detallada realizada.

muestran en la fig. 8. Se observa una gran coincidencia entre ambos.

Al contrario que en el experimento anterior, no se observan variaciones en la forma de los espectros con la modificación de los parámetros de simulación mixta excepto para $0^{\circ}$ cuando $C_{1}=C_{2}=0.2$. De nuevo esta discrepancia se explica por una diferencia en la fluencia energética de fotones.

Los efectos en la fluencia energética con la variación de los parámetros de simulación mixta se muestran en la fig. 9. No se observan diferencias estadísticamente significativas excepto en el eje central para el valor máximo de los parámetros $C_{1}$ y $C_{2}$ donde es un $4.2 \%$ inferior respecto al valor experimental. Los resultados de fluencia energética son estadísticamente equivalentes para valores iguales o menores a $C_{1}=C_{2}=0.1$.

De los resultados obtenidos se infiere que en la reproducción de este experimento se puede realizar una selección de los valores de los parámetros de simulación mixta más elevados sin correr el riesgo de obtener resultados inexactos en la simulación.

Los resultados (fig. 9) también sugieren, en comparación con los obtenidos en el experimento electrones de $15 \mathrm{MeV}$ en plomo (fig. 6), que la energía inicial de

Tabla 3. Valores relativos de eficiencia de las distintas simulaciones respecto a la simulación más detallada realizada $\left(C_{0.01}\right)$ para electrones de $200 \mathrm{keV}$ en $\mathrm{Al}$.

\begin{tabular}{c|c}
$\boldsymbol{C}_{\mathbf{1}}$ y $\boldsymbol{C}_{\mathbf{2}}$ & Eficiencia relativa \\
\hline 0.001 & 1 \\
\hline 0.05 & 4 \\
\hline 0.1 & 7 \\
\hline 0.2 & 10
\end{tabular}




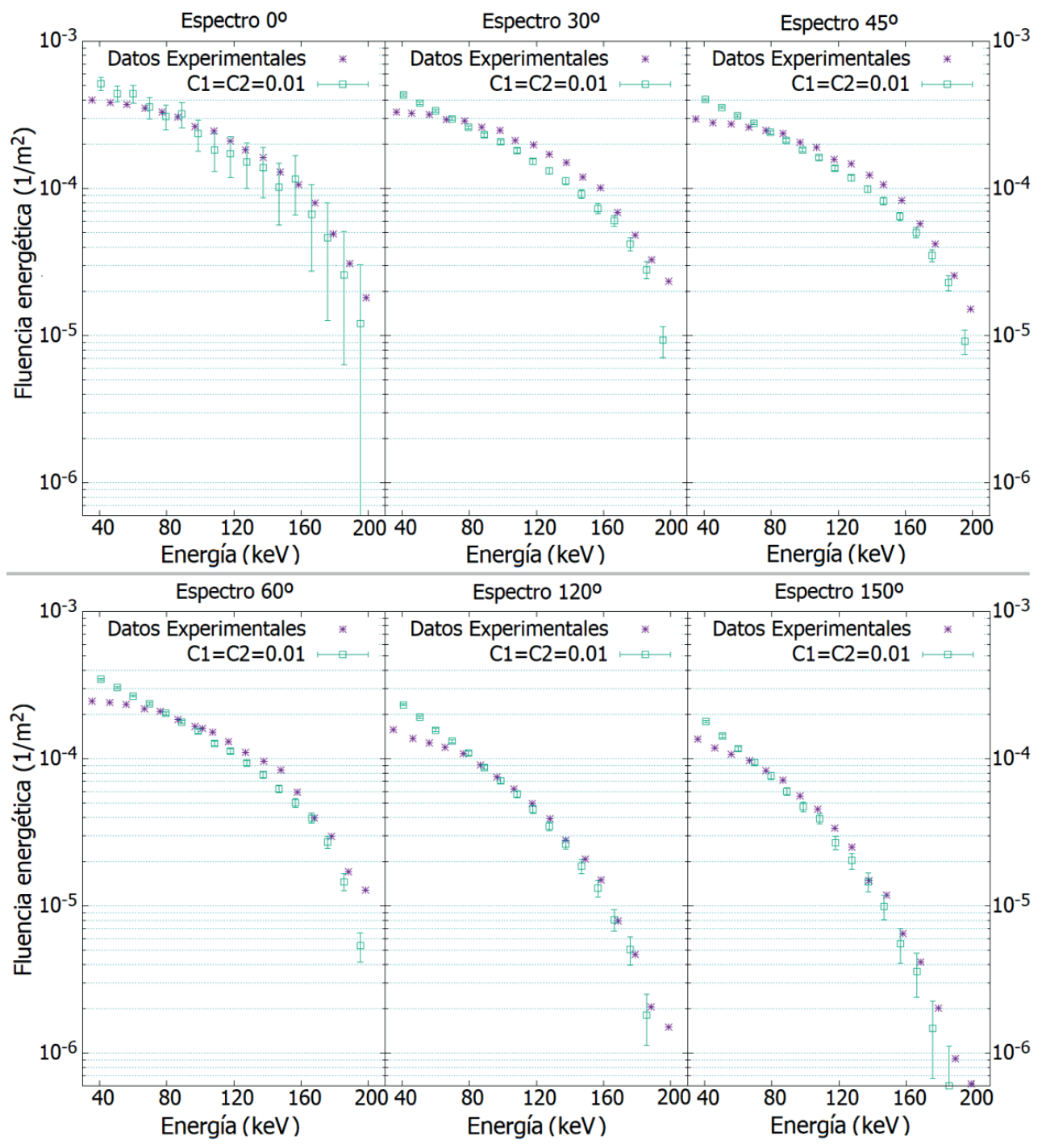

Fig. 7. Fluencia energética a 1 metro de fotones de bremsstrahlung para un haz de 200 keV sobre un blanco de Al y los ángulos de $0^{\circ}, 30^{\circ}, 45^{\circ}, 60^{\circ}, 120^{\circ}$ y $150^{\circ}$. Resultados experimentales y MC con los valores de simulación mixta $C_{1}=C_{2}=0.01$.

los electrones, el número atómico del material blanco elegido o una combinación de ambos son variables

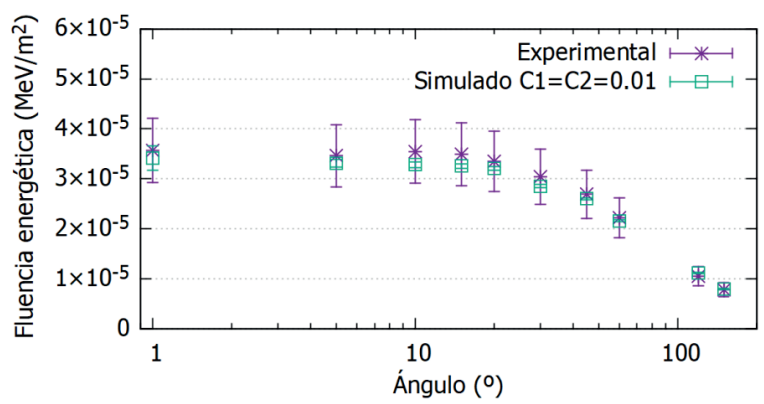

Fig. 8. Fluencia energética de fotones de bremsstrahlung para un haz de electrones de $200 \mathrm{keV}$ sobre un blanco de $\mathrm{Al}(\mathrm{Z}=13)$. dependientes en la correcta elección de los parámetros de simulación mixta.

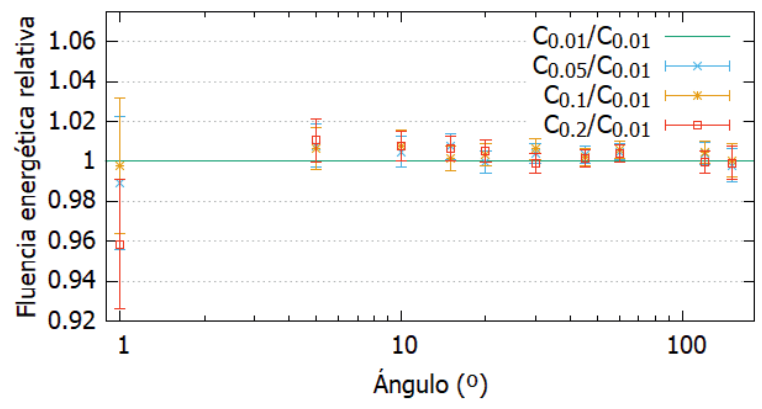

Fig. 9. Cociente entre el resultado de fluencia energética en cada ángulo en una simulación respecto al resultado obtenido con la simulación más detallada realizada. 
En la tabla 3 se muestra la eficiencia (1) relativa al variar $C_{1}$ y $C_{2}$. De nuevo se observa un notable aumento en el tiempo necesario de simulación para el mismo número de historias al reducir los parámetros de simulación mixta.

\section{Discusión}

En este trabajo se ha demostrado que una inadecuada elección de los parámetros de simulación mixta $C_{1}$ y $C_{2}$ ocasiona desviaciones en las fluencias obtenidas respecto a los valores experimentales.

Existe mucha bibliografía en el ámbito de la radioterapia en la que se reproducen propiedades de los haces simulando el campo de radiación generado mediante un haz de electrones impactando en un blanco grueso. Si se comparan los parámetros de simulación escogidos en este trabajo con los empleados por los diferentes autores se pueden obtener algunas conclusiones.

En la reproducción de fluencias, existen publicaciones con las que comparar los resultados experimentales de este trabajo. González et al. ${ }^{16}$ consiguen reproducir los resultados experimentales de un acelerador Elekta Precise dando unos valores de los parámetros de simulación mixta de 0.01 en el blanco de tungsteno (W) con $Z=74$, lo que coincide con los resultados de este trabajo.

Sempau et al. ${ }^{17}$ también obtienen buena concordancia con los resultados experimentales con un valor relativamente alto de los parámetros de simulación mixta $C_{1}=C_{2}=0.1$ si se comparan con los resultados de este trabajo. En este caso se ha hecho uso de Interaction Forcing, lo que sugiere que el uso de esta técnica de reducción de varianza podría estar facilitando resultados más exactos a pesar de elegir unos parámetros de simulación altos.

En el campo del radiodiagnóstico Gallardo et al. ${ }^{18}$ reproducen espectros con unos valores de $C_{1}=C_{2}=$ 0.2 . Estos valores de los parámetros de simulación mixta son relativamente altos comparados con los resultados obtenidos en este trabajo, sin embargo esto puede estar justificado por el mayor número de variables disponibles para la sintonización del haz primario o porque los espectros obtenidos se generan con un ángulo anódico superior a los $10^{\circ}$ de forma que las desviaciones en la fluencia son menos evidentes tal y como se muestra en este trabajo. Es por tanto lógico encontrar en la bibliografía simulaciones de haces de frenado para radiodiagnóstico con valores altos de $C_{1}$ y $C_{2}$.

En la simulación se puede recurrir a altos valores de los parámetros de simulación mixta, teniendo en cuenta siempre los efectos de sintonización del haz o del empleo de técnicas de reducción de varianza.
En caso de no emplear estas técnicas, el uso de altos valores de $C_{1}$ y $C_{2}$ puede conducir a resultados inexactos de fluencia tal y como se ha mostrado en este trabajo.

\section{Conclusiones}

Mediante simulaciones MC se pueden reproducir resultados experimentales de producción de fotones de bremsstrahlung con una gran precisión, sin embargo la exactitud de los resultados simulados es dependiente de una correcta elección de los parámetros de simulación mixta $C_{1}$ y $C_{2}$.

Se ha demostrado que altos valores de los parámetros de simulación mixta, especialmente a altas energías, conllevan una reproducción inexacta de características fundamentales del haz de radiación de frenado como son el espectro y la fluencia.

Valores muy bajos de estos parámetros aseguran la reproducción de los resultados experimentales a costa de un gran aumento en el tiempo necesario de simulación, pudiendo llegar a simulaciones inviables en la práctica. Existe un valor a partir del cual al disminuir el valor de los parámetros de simulación mixta no se obtienen variaciones estadísticamente significativas en los resultados.

Con electrones de alta energía impactando en blancos de número atómico alto, se necesita un mayor grado de detalle en la simulación $\left(C_{1}=C_{2}=0.01\right)$ para la reproducción de resultados experimentales que para electrones de baja energía en materiales de bajo número atómico $\left(C_{1}=C_{2}=0.1\right)$, por lo que la energía inicial del haz de electrones, el número atómico del material blanco o una combinación de ambos son variables que han de ser tenidas en cuenta a la hora de realizar una correcta selección de los parámetros de simulación mixta.

\section{Agradecimientos}

Este trabajo ha sido financiado en parte por la Junta de Andalucía (FQM0387), por los proyectos PI-0505-2017 y B-TIC-468-UGR18, y por el Fondo Europeo de Desarrollo Regional.

\section{Bibliografía}

1. Baró J, Sempau J, Fernández-Varea JM, Salvat F. An algorithm for Monte Carlo simulation of the penetration and energy loss of electrons and positrons in matter. Nucl Instr and Meth in Phys Res B 1995;100:31-46.

2. Faddegon BA, Asai M, Perl J, Ross C, Sempau J, Tinslay J, Salvat F. Benchmarking of Monte Carlo simulation of brem- 
sstrahlung from thick targets at radiotherapy energies. Med Phys 2008;35:4308-17.

3. Rodriguez M, Sempau J, Brualla L. Technical Note: Study of the electron transport parameters used in PENELOPE for the Monte Carlo simulation of Linac targets. Med Phys 2015;42:2877-81.

4. Pandola L, Andenna C, Caccia B. Validation of the Geant4 simulation of bremsstrahlung from thick targets below 3 MeV. Nucl Instr and Meth in Phys Res B 2015;350:41-8.

5. DeMarco JJ, Solberg TD, Wallace RE, Smathers JB. A verification of the Monte Carlo code MCNP for thick target bremsstrahlung calculations. Med Phys 1995;22:11-6.

6. Salvat F, Fernández-Varea JM, Sempau J, Llovet X. Monte Carlo simulation of bremsstrahlung emission by electrons. Radiat Phys Chem 2006;75:1201-19.

7. Seltzer SM, Berger MJ. Bremsstrahlung spectra from electron interactions with screened atomic nuclei and orbital electrons. Nucl Instr and Meth in Phys Res B 1985;12:95134.

8. Seltzer SM, Berger MJ. Bremsstrahlung energy spectra from electrons with kinetic energy $1 \mathrm{keV}-10 \mathrm{GeV}$ incident on screened nuclei and orbital electrons of neutral atoms with Z=1-100. Atom Data Nucl Data 1986;35:345-418.

9. Kissel L, Quarles CA, Pratt RH. Shape functions for atomicfield bremsstrahlung from electrons of kinetic energy 1-500 $\mathrm{keV}$ on selected neutral atoms $1 \leq \mathrm{Z} \leq 92$. Atom Data Nucl Data 1983;28:381-460.

10. ICRU Report 77. Elastic scattering of electrons and positrons. Journal of the International Commission on Radiation Units and Measurements 2007;7:1-162.
11. Salvat F. PENELOPE-2014: A Code System for Monte Carlo Simulation of Electron and Photon Transport. OECD Nuclear Energy Agency, Issy-les-Moulineaux, France, 2015.

12. Faddegon BA, Ross CK, Rogers DWO. Forward-directed bremsstrahlung of 10-30 MeV electrons incident on thick targets of Al and Pb. Med Phys 1990;17:773-85.

13. Faddegon BA, Ross CK, Rogers DWO. Angular distributions of bremsstrahlung from $15 \mathrm{MeV}$ electrons incident on thick targets of Be, Al and Pb. Med Phys 1991;18:727-39.

14. Rester DH, Dance WE, Derrickson JH. Thick target Bremsstrahlung Produced by electron bombardment of Targets of Be, Sn and Au in the energy range 0.2-2.8 MeV. J Appl Phys 1970;41:2682-92.

15. Dance WE, Rester DH, Farmer BJ, Johnson JH. Bremsstrahlung produced in thick aluminium and iron targets by 0.5 to $2.8 \mathrm{MeV}$ electrons. J Appl Phys 1968;39:28819.

16. González W, Lallena AM, Alfonso R. Monte Carlo simulation of the dynamic micro-multileaf collimator of a LINAC Elekta Precise using PENELOPE. Phys Med Biol 2011;56:3417-31.

17. Sempau J, Badal A, Brualla L. A PENELOPE-based system for the automated Monte Carlo simulation of clinacs and voxelized geometries-application to far-from-axis fields. Med Phys 2011;38:5887-95.

18. Gallardo S, Querol A, Pozuelo F, Verdú G, Ródenas J. Application of the Monte Carlo codes PENELOPE and MCNP5 to unfold X-ray spectra in the diagnostic energy range. Radiat Phys Chem 2014;95:166-9. 


\section{SIEMENS}

\section{Small cells don't have to lead to big problems.}

Siemens answers help doctors detect diseases earlier, saving costs and extending lives.

When diseases are caught early, they make less of an impact on everyone. That's because a large majority of today's healthcare costs go toward treating the late stages of diseases like cancer and heart disease. With Siemens advanced diagnostic technology, doctors can accurately identify these killers earlier. So patients get the treatment they need sooner. Which helps save lives and cuts costs. Wherever there are tough healthcare questions, we're answering them. 